\title{
Observations of IMF coherent structures and their relationship to SEP dropout events
}

\author{
L. Trenchi ${ }^{1}$, R. Bruno ${ }^{1}$, R. D'Amicis ${ }^{1}$, M. F. Marcucci ${ }^{1}$, and D. Telloni ${ }^{2}$ \\ ${ }^{1}$ INAF - Istituto di Astrofisica e Planetologia Spaziali, Via Fosso del Cavaliere 100, 00133 Rome, Italy \\ ${ }^{2}$ INAF - Osservatorio Astronomico di Torino, Via dell'Osservatorio 20, 10025 Pino Torinese, Italy \\ Correspondence to: R. Bruno (roberto.bruno@iaps.inaf.it)
}

Received: 28 March 2013 - Revised: 19 June 2013 - Accepted: 24 June 2013 - Published: 6 August 2013

\begin{abstract}
The solar energetic particle (SEP) events from impulsive solar flares are often characterized by short-timescale modulations affecting, at the same time, particles with different energies. Several models and simulations suggest that these modulations are observed when SEPs propagate through magnetic structures with a different connection with the flare site. However, in situ observations rarely showed clear magnetic signatures associated with these modulations.

In this paper we used the Grad-Shafranov reconstruction to perform a detailed analysis of the local magnetic field topology during the SEP event of 9-10 January 1999, characterized by several SEP dropouts. An optimization procedure is used to identify, during this SEP event, the magnetic structures which better satisfy the Grad-Shafranov assumptions and to evaluate the direction of their invariant axis.

We found that these two-dimensional structures, which are flux ropes or current sheets with a more complex field topology, are generally associated with the maxima in the SEP counts. This association suggests that the SEPs propagate within these structures and, since their gyration radii is much smaller than the transverse dimension of these structure, cannot escape from them.
\end{abstract}

Keywords. Interplanetary physics (Energetic particles; interplanetary magnetic fields; solar wind plasma)

\section{Introduction}

Solar energetic particle (SEP) events originate during the explosive release of magnetic energy in solar flares or ahead of coronal mass ejections. These energetic particles, whose energy ranges from 0.1 to $1000 \mathrm{MeV} \mathrm{amu}^{-1}$, and even more once they have been released, propagate along interplanetary magnetic field (IMF) lines. The classical picture (see references in Reames, 1999) considers two different acceleration processes.

At the flare site - the source of explosive events which follow sudden changes of magnetic field strength or configuration due to large magnetic loop structures reconnection caused by the motion of their footpoints on the solar surface - particles can be accelerated in a single step (a few tens of seconds) via resonant stochastic acceleration. This kind of event, generated by a localized source (flare), is dominated by electrons and it shows $\sim 1000$-fold enhancements in ${ }^{3} \mathrm{He} /{ }^{4} \mathrm{He}$ and $\sim 10$-fold enhancements in $\mathrm{Fe} / \mathrm{O}$ (Reames, 1994).

In addition, another possible mechanism to produce an SEP event is based on the interaction between the outward propagating shock front preceding the CME and the solar wind, and it is referred to as first-order Fermi acceleration. In this case, particles repeatedly moving from upstream to downstream and vice versa through the shock front continuously gain energy until they have enough energy to escape from the shock. This kind of event, generated by an extended source region (shock in front of the CME), shows the most intense ion fluxes, the highest energies and an enhanced electrons to protons ratio.

Particles generated by an impulsive solar flare are characterized by a clear velocity dispersion due to the fact that the acceleration time is much less that the travel time to the observer, in our case $1 \mathrm{AU}$. The length of the SEPs' interplanetary travel paths can be estimated by studying the arrival times when the first particles, at different energies, are measured by the detector. Masson et al. (2012) proposed a new normalized method to perform this analysis, reducing the effect of energetic particle background. 
About half of impulsive SEP events show clear dropouts (Chollet and Giacalone, 2008), limited by sharp boundaries, affecting all energies at the same time. These events are characterized by a noticeable decrease in flux intensity and very sharp edges of duration of the order of a few minutes. These features suggest that the origin of these voids should be sought in the interplanetary space rather than at the flare site. Although there is unanimous consensus about this conclusion, there is not an equivalent agreement on the physical mechanisms at the origin of these dispersionless events.

Mazur et al. (2000) analysed different SEP events characterized by clear dropouts, but concluded that these events did not correlate with large changes in the direction of the local interplanetary magnetic field. They proposed a scenario in which, during the wind expansion to $1 \mathrm{AU}$, the footpoints of flux tubes connected to a given active region become mixed with the footpoints of other flux tubes that are magnetically disconnected from that same region because of the convective motion at the solar surface. When the flare occurs, energetic particles will be channelled only along those tubes magnetically connected to the flare site. Because of the shuffling of these footpoints, flux tubes that are contiguous at 1 AU might originate from different regions of the Sun. As a consequence, contiguous flux tubes at $1 \mathrm{AU}$ could be alternatively filled by energetic particles or be empty, depending on whether or not they are magnetically connected to the flare region. Thus, an observer going through these different and contiguous flux tubes would observe the typical signature of SEP dropouts. However, we remark that these authors did not find any correlation between the local interplanetary magnetic field and the clear dropouts observed in the energetic particles flux intensity.

In order to explain the observations by Mazur et al. (2000), Giacalone et al. (2000) performed numerical simulations of the propagation of solar energetic particles in a turbulent magnetic field environment, similar to that observed in the solar wind. They showed that, if the source region that releases impulsively the energetic particles is small compared to the field correlation scale, we should expect to observe modulations of the intensity flux associated with remarkably steep spatial gradients, very similar to what has been observed at $1 \mathrm{AU}$. The reason resides in the combined effect of random walk of interplanetary magnetic field lines due to turbulence and the subdiffusive regime of perpendicular motion with respect to the local magnetic field line. Furthermore, the same authors showed that no dropouts are observed when the injection region is rather large, i.e. several magnetic field correlation scales.

Other authors, like Ruffolo et al. (2003), Pommois et al. (2005), Chuychai et al. (2007), Qin and Li (2008), Zimbardo et al. (2008), Dröge (2003) and Dröge and Kartavykh (2009), among others, addressed the specific role of turbulence in SEP modulation attributing a different role to SLAB and 2-D components.
In particular, according to Ruffolo et al. (2003), dropouts can be identified with topological structures that develop during the evolution of the solar wind turbulence and do not depend on the initial motions at the solar surface. In their 2D+SLAB model, particles are injected from a localized source region at the Sun. During the wind expansion into the interplanetary space, while some field lines form coherent small-scale helical filaments identifiable as 2-D structures, other field lines governed by SLAB turbulence spread aside, following a random walk. Since lateral diffusion coefficient is much less than parallel coefficient, SEPs remain tightly connected to magnetic field lines which diffuse differently depending on 2-D or SLAB dominance, and this leads to observe regions of high SEP flux interleaved by extended regions of very low flux (dropouts).

An opposite role of 2-D and SLAB turbulence in SEP modulation was suggested by Pommois et al. (2005) and Zimbardo et al. (2008). In the paper by Pommois et al. (2005) these authors modelled the energetic particles' transport in a magnetic field configuration due to the background field plus magnetic turbulence. The 3-D simulation box was filled with static turbulence (2D+SLAB) whose anisotropy could be changed from the isotropic case where $\ell_{\perp}=\ell_{/ /}$to the quasi-2-D case with $\ell_{\perp}<\ell_{/ /}$or to the quasi-slab case $\ell_{\perp}>\ell_{/ /}$, where $\ell_{\perp}$ and $\ell_{/ /}$are the perpendicular and parallel correlation lengths of the magnetic field turbulence, respectively. In this configuration, particles travel across a number of correlation lengths equivalent to the real path between the Sun and Earth, and these authors found that transport parallel and perpendicular to the ambient mean field is strongly influenced by the ratio $\ell_{\perp} / \ell_{/ /}$. They showed that a modulation of the SEP intensity very similar to the one observed during dropout events could be recovered for $\ell_{\perp}>\ell_{/ /}$, i.e. for a turbulence dominated by the SLAB component. In fact, the SEPs would perform most of their interplanetary path in a slab-like spectral turbulence, since this kind of turbulence is expected to be dominant in the inner heliosphere (Ruiz et al., 2011).

Chollet and Giacalone (2008) noticed that SEP dropouts from impulsive events most likely arise from the crossing of the observer through alternating filled and empty tubes of energetic particles arising from the random walk of field lines directly connected to or disconnected from the source region at the Sun. These authors found that ACE and WIND spacecraft observed different features in the flux of the energetic particles when they were separated in heliographic longitude by more than the local turbulence correlation scale, i.e. $\sim 10^{6} \mathrm{~km}$. However, they also noticed that these events did not seem to be systematically associated with changes in the magnetic field, and the possible association was due to random coincidence. However, since some dropouts have been associated by Gosling et al. (2004) to changes in the intensity of the solar wind electron strahl, the same authors suggested to perform further studies in order to better understand the 
role of field line mixing on the modulation of the flux of energetic solar particles.

This suggestion was followed in a recent paper by Trenchi et al. (2013), where the authors performed a detailed analysis of the local magnetic field and plasma parameters measured in situ during impulsive SEP events in order to search for a possible association between clear SEP dispersionless modulations and changes in the solar wind parameters. These authors identified magnetic helicity signatures and found convincing evidence for their association with the borders of impulsive SEP events or with SEP dropout events. The same authors suggested the idea that these magnetic coherent structures might be thought to be the borders between adjacent magnetic flux tubes (Bruno et al., 2001; Li, 2007, 2008; Borovsky, 2008). This conclusion is not in contrast with previous models which suggested the idea of field lines either connected to or disconnected from the flare site (Mazur et al., 2000; Chollet and Giacalone, 2008).

The present paper, which follows at closely behind the paper by Trenchi et al. (2013), is a further study on the magnetic field topology in close association with the presence of SEP dropouts. In particular, we attempted to reconstruct the 2-D magnetic topology of coherent magnetic structures identified during these events. To do so we adopted the Grad-Shafranov (GS) reconstruction, first developed by Sonnerup and Guo (1996) to study the structure of magnetopause current layer (see Appendix A for details on this method), which has been successfully applied to a variety of coherent magneto-hydrostatic structures observed in the solar wind, such as magnetic clouds (Hu and Sonnerup, 2002), small-scale magnetic flux ropes ( $\mathrm{Hu}$ and Sonnerup, 2001) and multiple magnetic flux ropes (Hu et al., 2003, 2004). This technique is particularly useful since it can be applied to any quasi-stationary two-dimensional magnetic field structure, without further assumptions on its magnetic topology. With data collected by a single spacecraft, the GS reconstruction produces magnetic field maps which give a direct visualization of the magnetic field configuration in extended regions near the spacecraft. In the following section of the present paper we will show how this technique is able to identify and reconstruct 2-D structures directly connected to SEP dropout events during the 9-10 January 1999 event recorded by ULEIS/ACE.

\section{Data analysis}

Figure 1 illustrates the SEP events detected by ACE during the 9-10 January 1999 event (adapted from Trenchi et al., 2013). The energetic ion data, in the energy range from 0.014 to $1.42 \mathrm{MeV}$ nucleon $^{-1}$, were obtained from the Ultra Low Energy Isotope Spectrometer (ULEIS) (Mason et al., 1998). The spectrogram in panel (a) shows the energy (in units of $\mathrm{MeV}$ nucleon ${ }^{-1}$ ) of energetic ions versus their arrival time, while panel (b) displays the energetic ion counts, integrated over all energies, vs. time, in about $5 \mathrm{~min}$ bins (adapted from the online plots available at http://www.srl.caltech.edu/ACE/ ASC/DATA/level3/uleis/).

The SEP event starts at about 15:00 UT on 9 January with the arrival of ions with energy of about $0.4 \mathrm{MeV}_{\text {nucleon }}{ }^{-1}$, finishes approximately at 05:30 on 10 January and shows a clear dispersion relation, which suggests that the particles are accelerated impulsively at the Sun (Mazur et al., 2000; Gosling et al., 2004). In fact, for this SEP event, the slope of the dispersion relation in the $1 / V$ spectrogram (available at http://www.srl.caltech.edu/ACE/ASC/DATA/level3/uleis/) suggests a length of the interplanetary travel path of about 1.2 AU, which is similar to the one expected along the Parker spiral. Moreover, this event is characterized by several SEP modulations where the counts change, over timescales of about an hour, from the background level of about 50 particles / bin to more than 200 particles / bin.

In the next panels magnetic field and plasma data, obtained respectively from MAG (Smith et al., 1998) and SWEPAM (McComas et al., 1998), are reported. In particular, in panel (c) we show values of plasma $\beta$, while in panel (d) we show the azimuthal and polar angles of the magnetic field vector orientation in GSE coordinate system. The azimuthal angle varies between $-180^{\circ}$ and $+180^{\circ}$.

Trenchi et al. (2013) studied the magnetic field topology during several SEP events by evaluating the magnetic helicity, which is a physical descriptor that measures the twist of magnetic field lines. By adopting the use of the wavelet transform (Bruno et al., 2008; He et al., 2011; Podesta and Gary, 2011; Telloni et al., 2012), they studied the time behaviour of reduced magnetic helicity at different timescales. For this event, they found a rather complex field topology during these SEP modulations. Moreover, they noted that some of these SEP dropouts are associated with the high- $\beta$ regions, highlighted in Fig. 1 by the yellow-shaded areas.

In this work we take a step further in refining the analysis of magnetic field topology by using GS reconstruction. This technique, described in detail in the Appendix, is used to recover the coherent magnetic structures observed during this SEP event. Here, the deHoffmann-Teller (HT) analysis gives an excellent constant $V_{\mathrm{HT}}$, with a correlation coefficient among the electric fields $-V \times B$ and $-V_{\mathrm{HT}} \times B$ greater than 0.998 (not shown). This suggests that, in this data interval, magnetic structures approximately in a static equilibrium are advected with a constant speed through the spacecraft.

The optimization procedure illustrated by $\mathrm{Hu}$ and Sonnerup (2002) is used to identify the structures which better satisfy the GS assumptions. This procedure, originally developed to study the low- $\beta$ magnetic flux-ropes such as magnetic clouds, has been successfully applied to a number of structures in the solar wind, all characterized by $\beta<<1$ (Hu et al., 2003, 2004). Since, to our knowledge, the Hu and Sonnerup (2002) method has never been tested in the high- $\beta$ structures, we preferred to limit our analysis to the intervals 


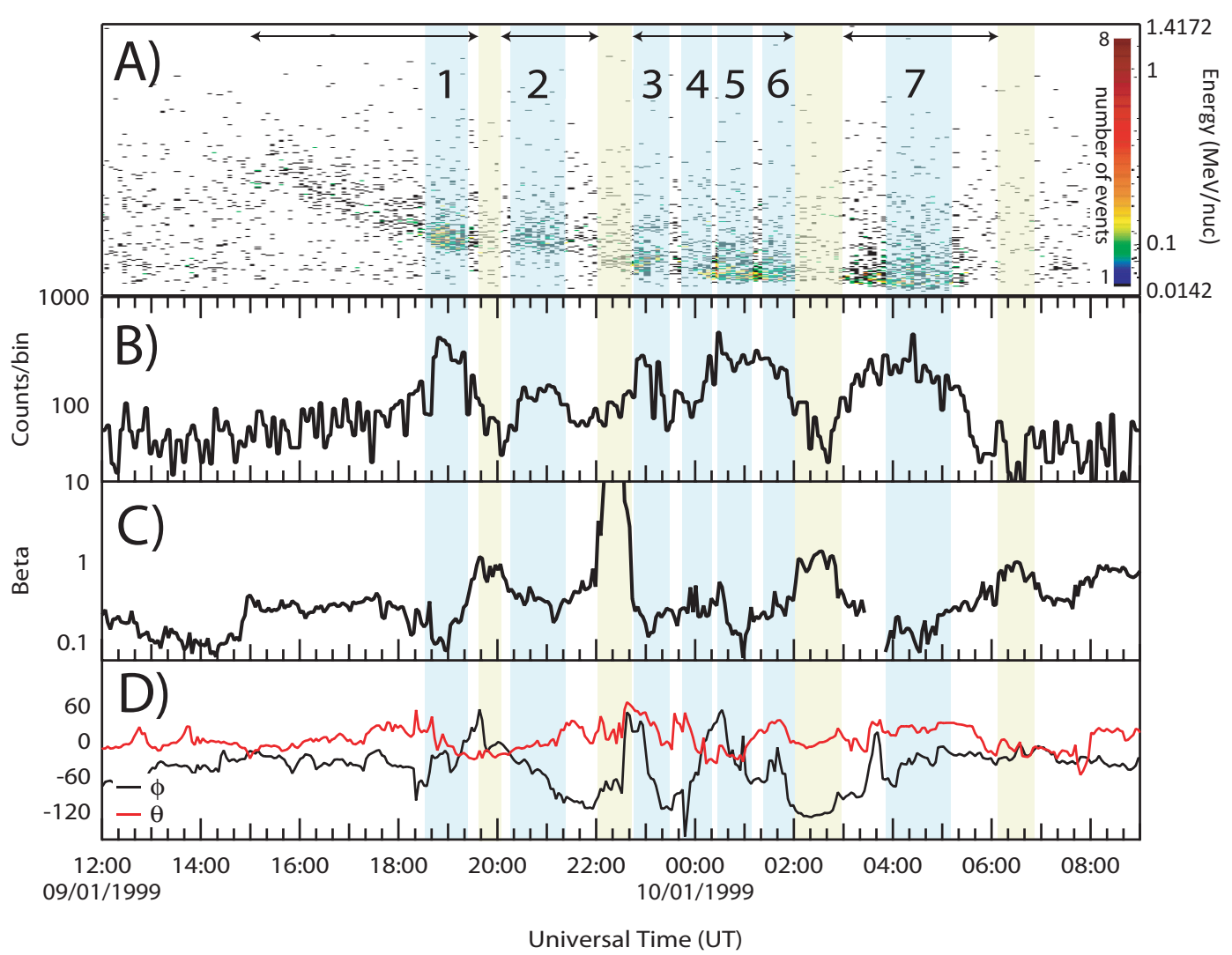

Fig. 1. Combined energetic ions and plasma-magnetic field parameters for the 9-10 January 1999 SEP event (adapted from Trenchi et al., 2013). In panel (a) the energy of ions (in units of $\mathrm{MeV}$ nucleon $^{-1}$ ) versus their arrival time; in panel (b) the ion counts, integrated over all energies, vs. time, in about 5 min bins. In panel (c) the ratio between plasma and magnetic pressures $\beta$, and in panel (d) the azimuthal and polar angles of the magnetic field vector, in GSE coordinate system, in the range $\left[-180^{\circ},+180^{\circ}\right]$ and $\left[-90^{\circ},+90^{\circ}\right]$, respectively. The blue-shaded areas highlight the structures reconstructed with the GS method.

where $\beta<1$, indicated with the black arrows on the top of Fig. 1, which cover most of the SEP event.

The application of the Hu and Sonnerup (2002) method on a certain data interval gives a residue RES (Eq. 5 of $\mathrm{Hu}$ and Sonnerup, 2002), which is proportional to the deviation of the structure from the GS assumptions. By performing this method on a sliding window, we identified the seven regions where the method gives the lower values of RES, highlighted by blue-shaded areas in Fig. 1. In these regions, the small dispersion of the transverse pressure $P_{\mathrm{t}}$ versus a singlevalue function of the $z$ component of the vector potential $A$, demonstrates that the observed structure satisfies with good approximation the GS assumptions, and the chosen $z$ axis is close to the real axis of the structure.

In Fig. 2 we show the results of the GS reconstruction for intervals 1 to 3 highlighted in Fig. 1. The left panel of each row shows the behaviour of $P_{\mathrm{t}}(A)$ (red line), the black line shows the behaviour of the third-order polynomials fits of $P_{\mathrm{t}}(A)$ used for the GS reconstruction and the blue lines represent the $95 \%$ confidence band. The fitting residual $R_{\mathrm{f}}$ (evaluated with Eq. A2 in the Appendix A), reported for each structure in the left panels, has been used by Hu et al. (2004) as a quantitative estimation of the deviation from the GS assumptions. Hu et al. (2004) assessed that the structures with $R_{\mathrm{f}} \leq 0.14$ satisfy well the two-dimensional equilibrium assumed by the GS equation. Therefore, we can consider that the structures reported in Fig. 2, which all have $R_{\mathrm{f}} \leq 0.14$, are properly recovered with the GS method.

On the right panel, the magnetic field maps obtained with the GS reconstructions, following the integration scheme illustrated by Hau and Sonnerup (1999), are reported. The equipotential lines of $A$, reported with the colour scale, represent the magnetic field lines in the reconstruction plane. The horizontal lines at the centre of the maps show the satellite path through these structures, whose dimensions are reported along the axes in $\mathrm{km}$. The black lines along the satellite path show the projection on the $x-y$ plane of the magnetic field vectors measured by the spacecraft along its trajectory (a scale corresponding to $5 \mathrm{nT}$ is reported).

We performed the reconstructions within a rectangular box with a small aspect ratio $\left(\frac{y}{x}=\frac{1}{5}\right)$ in order to limit the numerical instabilities which develop at larger $y$. Moreover, the 
1) $18: 31-19: 23$ on 09 January
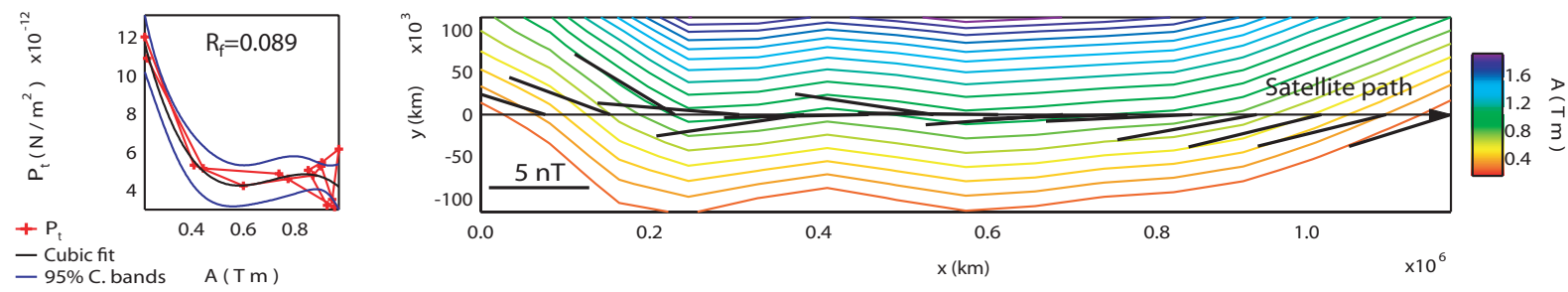

2) $20: 13-21: 21$ on 09 January
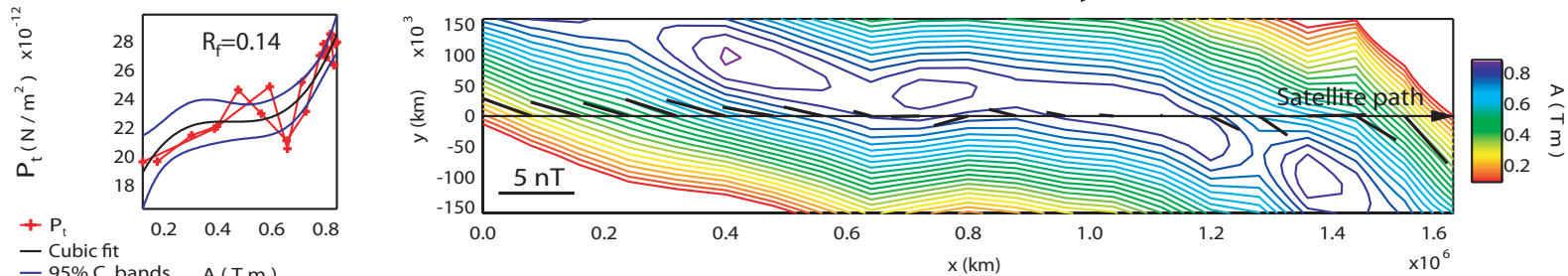

3) 22:43-23:31 on 09 January

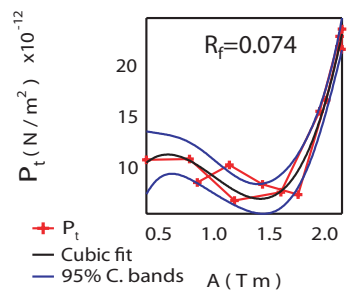

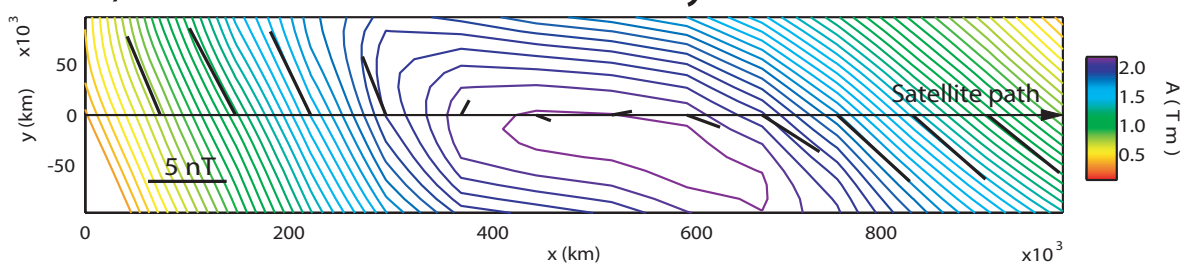

Fig. 2. The results of Grad-Shafranov reconstruction for the structures highlighted with blue-shaded areas in Fig. 1. The left panels illustrate the behaviour of $P_{\mathrm{t}}(A)$ (red line), the black lines report the third-order polynomials fits of $P_{\mathrm{t}}(A)$ used for the GS reconstruction and the blue lines represent the $95 \%$ confidence bands of the fit. The fitting residual $R_{\mathrm{f}}$ is a quantitative estimation of the deviation from the model assumptions. In the right panels, the magnetic field maps obtained with the GS reconstructions are reported. The equipotential lines of $A$, reported with the colour scale, represent the magnetic field lines in the reconstruction plane. The horizontal lines at the centre of the maps show the satellite path through these structures, whose dimensions are reported along the axes in $\mathrm{km}$. The black lines along the satellite path show the projection on the $x-y$ plane of the magnetic field vectors measured by the spacecraft along it trajectory (a scale corresponding to $5 \mathrm{nT}$ is reported).

reconstruction is completed only in the portion of the reconstruction plane magnetically connected to the spacecraft trajectory; that is, in the region where $A$ is within the $A$ range obtained along the $x$ axis. In this way, we did not perform extrapolations of $P_{\mathrm{t}}(A)$, which could introduce uncertainties in the reconstruction maps.

The reconstruction of structure 1, observed around 19:00 on 9 January (Fig. 2, panel 1), shows several open field lines which are bent upward. The shape of these field lines would suggest the presence of a magnetic island whose centre should be somewhere beyond $10^{5} \mathrm{~km}$ in the positive $y$ direction. However, since these field lines go beyond the reconstruction plane, it is not possible to infer the exact magnetic topology of this structure. The structure 2, observed around 20:40 on 9 January (Fig. 2, panel 2), seems to be a current sheet characterized by a string of magnetic islands separating two regions of opposite field polarity. These islands have the same chirality (same sign of vector potential associated with unidirectional $B_{\mathrm{Z}}$ component), and therefore they are separated by $x$ points. The structure 3, observed around 23:00 on 9 January (Fig. 2, panel 3), is clearly a flux rope, being characterized by several closed field lines in the reconstruction plane, elongated in the $x$ direction. Toward the centre of this structure the axial magnetic field increases, while the plasma pressure decreases (not shown). This configuration results, in the three-dimensional space, in nested helical field lines. It can be noted that each of these structures is approximately overlapped with a peak in the SEP counts (Fig. 1).

In Fig. 3 results of the GS reconstruction, for intervals 4 to 7 highlighted in Fig. 1, are illustrated. The format is the same as Fig. 2. The structures 4 to 6 , which have $R_{\mathrm{f}} \leq 0.13$, are properly recovered with the GS method. Conversely, the larger value of $R_{\mathrm{f}}$ for the structure 7 could indicate deviations from the two-dimensional equilibrium.

In structure 4, detected around 00:00 on 10 January (Fig. 3, panel 1), a closed magnetic island is observed along the 

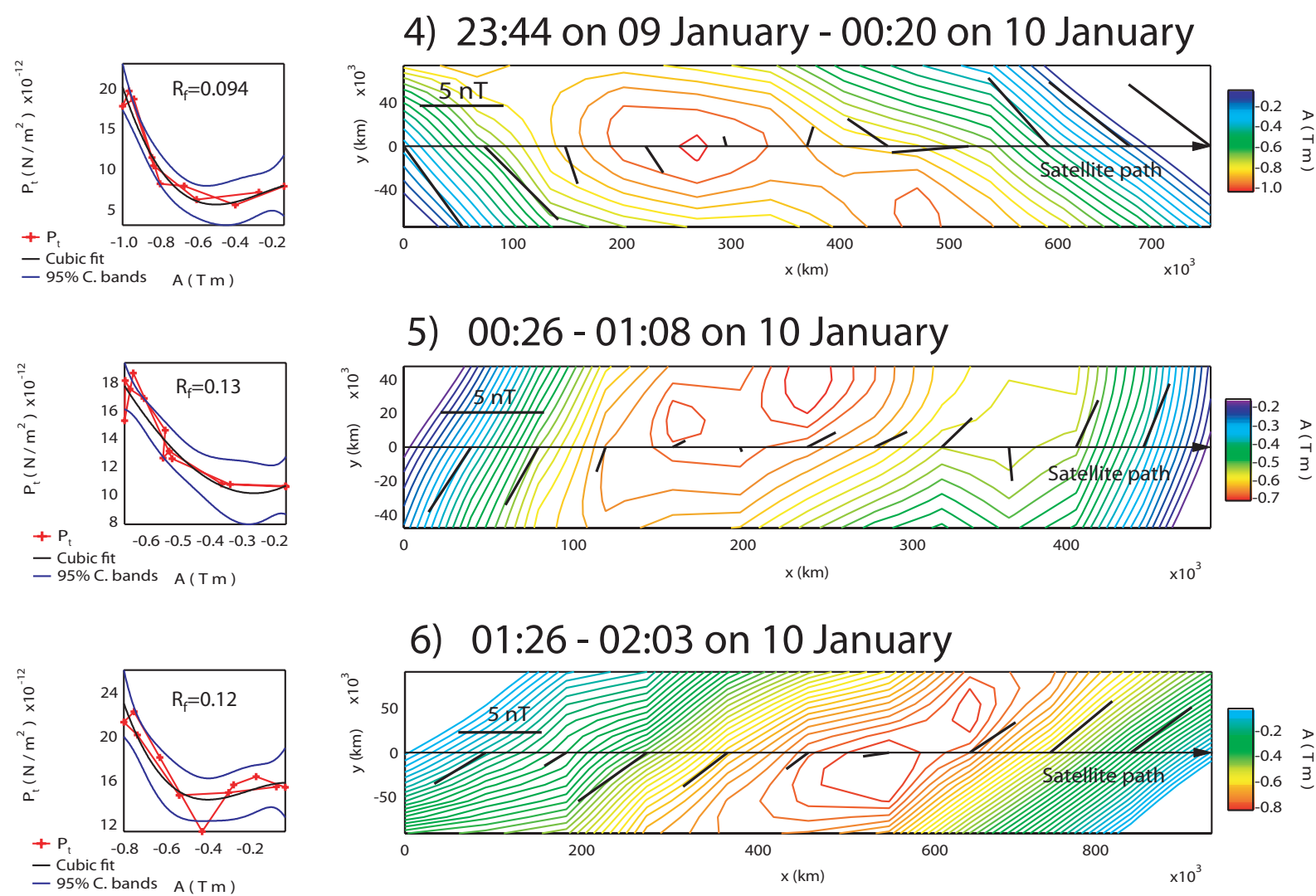

6) 01:26 - 02:03 on 10 January

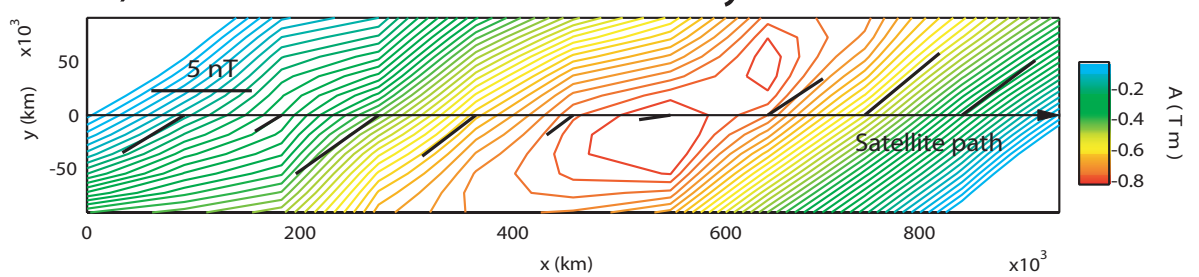

7) $03: 51-05: 11$ on 10 January
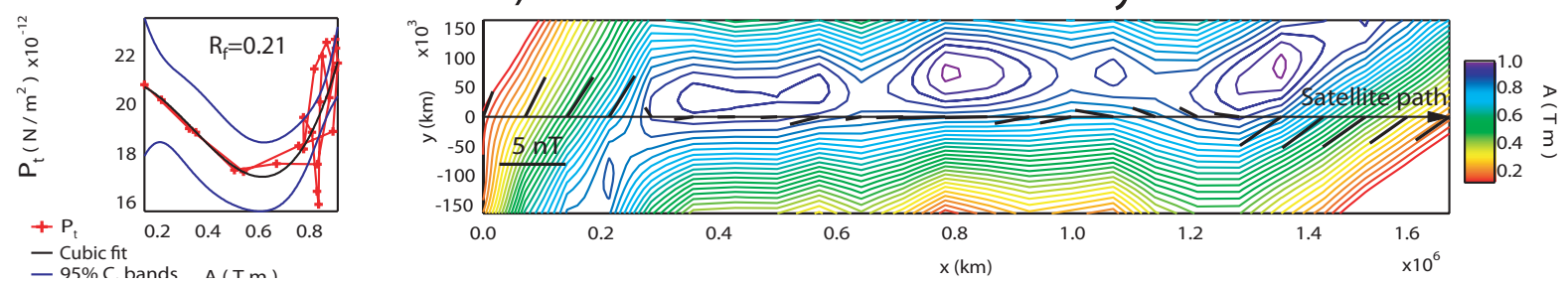

Fig. 3. The results of Grad-Shafranov reconstruction for the structures highlighted with blue-shaded areas in Fig. 1. The format is the same as Fig. 2.

spacecraft trajectory, on the left of the map. Moreover, another island is partially recovered on the bottom right part of the map and perhaps another one on the top left corner of the map. These islands are well separated, and they are embedded within oppositely oriented magnetic fields. Therefore this structure is probably part of a current sheet, extended outside of the reconstruction plane, similar to one recovered in structure 2. However, in the structure 5 (Fig. 3, panel 2), the two islands recovered at the centre of the structure are embedded within closed field lines, which are bent toward the centre of the structure. Therefore this structure could be considered as a flux rope, or rather as a double flux rope, similar to the ones observed by $\mathrm{Hu}$ et al. (2004). A similar topology is recovered for structure 6, observed around 01:45 on 10 January (Fig. 3, panel 3). During these structures, a single SEP intensification is observed - structure 4 during the increase of SEP counts and structures 5 and 6 during the peak of SEP counts.

In structure 7 (Fig. 3, panel 4), the spacecraft goes across a current sheet, where a string of magnetic islands, in some cases inside larger islands, are observed. This structure matches the SEP intensification observed after 03:00 on 10 January, even if its time duration is shorter. However, it should be noted that this GS reconstruction started at 03:51 on 10 January because of a gap in the plasma data from 03:27 to $03: 50$.

\section{Conclusions}

In this paper we studied the local magnetic field topology during the SEP dropout events of 9-10 January 1999 
Table 1. The table reports the principal characteristics of the seven structures recovered with GS method. The start and end times, their field topology (current sheet CS), flux rope (FR) or uncertain topology (?)), their average $\beta$ values and their quality factor for GS assumptions $R_{\mathrm{f}}$ are reported in the first four columns. Then, it is reported the direction of the invariant axis of these structures, with the azimuthal and polar angles, and the average SEP counts measured within these structures.

\begin{tabular}{ccccccc}
\hline Structure \# & Time interval & Field topology & $\beta$ & $R_{\mathrm{f}}$ & Axis orientation & Avg. SEP counts \\
\hline 1 & 18:31-19:23, 9 Jan & $?$ & 0.166 & 0.089 & $\left(-8^{\circ}, 48^{\circ}\right)$ & 271 \\
2 & 20:13-21:21,9 Jan & CS & 0.341 & 0.14 & $\left(-8^{\circ}, 113^{\circ}\right)$ & 119 \\
3 & 22:43-23:31,9 Jan & FR & 0.217 & 0.074 & $\left(25^{\circ}, 125^{\circ}\right)$ & 178 \\
4 & 23:44 9 Jan-00:20 10 Jan & CS & 0.290 & 0.094 & $\left(-8^{\circ}, 123^{\circ}\right)$ & 140 \\
5 & 00:26-01:08, 10 Jan & FR & 0.223 & 0.13 & $\left(-22^{\circ}, 162^{\circ}\right)$ & 288 \\
6 & 01:26-02:03, 10 Jan & FR & 0.309 & 0.12 & $\left(24^{\circ}, 109^{\circ}\right)$ & 189 \\
7 & 03:51-05:11, 10 Jan & CS & 0.142 & 0.21 & $\left(29^{\circ}, 128^{\circ}\right)$ & 217 \\
\hline
\end{tabular}

recorded by the ACE/ULEIS experiment (Mason et al., 1998). To do so we adopted the Grad-Shafranov technique, which allows for the reconstruction of 2-D magnetohydrostatic structures that are passively advected past the observer. This technique (Sonnerup and Guo, 1996) proved to be successful in reconstructing the magnetic field structure in a variety of cases, including magnetic clouds, flux ropes and multiple flux ropes (Hu and Sonnerup, 2001, 2002; Hu et al., 2003, 2004).

The SEP event considered in this paper shows several SEP modulations where the counts remarkably change over timescales of about an hour: the maximum counts are a few hundred particles / bin, while the minimum counts are comparable with the background level observed before and after the SEP event, which is approximately 50 particles / bin. In connection with these maxima we found two-dimensional magnetic structures, recovered with the GS method, whose main features are reported in Table 1. Some of them, like the events we indicated with numbers 3,5 and 6 , are magnetic flux ropes and have characteristics and size similar to the small-scale magnetic flux ropes frequently observed in the solar wind (Moldwin et al., 2000). Other structures, such as events 2 and 7, have a more complex topology that resembles a sort of current sheet with an embedded string of magnetic islands with the same chirality. However, the examination of plasma and magnetic field data suggests that these structures are not related with crossings of the heliospheric current sheet, which occurs a few days before and after this SEP event, approximately on 5 and 14 January 1999. However, in both cases field lines tend to remain in a nested configuration within each of these magnetic structures. Therefore the SEPs, which have a gyration radii much smaller than the transverse dimension of these structures, cannot escape from them (Krittinatham and Ruffolo, 2009).

This might be the key to explaining the enhancement of SEP counts if we adopt the suggestions given by Ruffolo et al. (2003) regarding the opposite role played by 2-D- and slab turbulence. As a matter of fact, while 2-D turbulence is characterized by field lines forming coherent small-scale helical filaments, in the slab component, field lines spread aside during the turbulence evolution. Pioneering works by Jokipii (1966) and Jokipii and Parker (1968) and the works by Giacalone and Jokipii (1999) and Giacalone et al. (2000) showed that the perpendicular transport strongly involves the contribution due to the random walk of the magnetic field lines due to turbulence. As a consequence, given that the parallel diffusion is much larger than the perpendicular one, we expect to have higher SEP fluxes during time intervals when turbulence is dominated by the 2-D component in the hypothesis that the field lines are connected to the flare site.

Whether these 2-D structures are formed during the turbulence evolution or rather that they are the interplanetary counterparts of magnetic structures already present at the flare site, like the magnetic flux tubes (Bruno et al., 2001), cannot be said. As a matter of fact, the hypothesis of flux tubes connected to or disconnected from the flare site being the main mechanism at the basis of SEP dropouts has been claimed in the literature (Mazur et al., 2000; Masson et al., 2012). Furthermore, the possibility that small-scale flux tubes are fossil structures that originate at the solar surface and that they might survive the dynamical expansion of the solar wind as far as $1 \mathrm{AU}$ (Thieme et al., 1989; Bavassano and Bruno, 1989; Tu and Marsch, 1990, 1993; Bruno et al., 2001 ) is supported by an exhaustive data analysis performed by Borovsky (2008). In particular, this author stressed the idea that the inner heliosphere is filled with a network of entangled magnetic flux tubes, and argued that the flux tubes do not reconnect during the advection time to $1 \mathrm{AU}$ owing to the expansion of the solar wind, during which these flux tubes are being pulled apart. In contrast, it seems that magnetic reconnection could occur at the front of the magnetic clouds, which are large-scale magnetic flux ropes moving faster than surrounding solar wind (Dasso et al., 2006; Ruffenach et al., 2012). In this case, particles travelling in magnetic clouds can escape from the eroded parts of these flux ropes.

In any case, our results highlight that the small-scale structures in the interplanetary medium can play an important role in the SEP modulations. Further studies are needed to investigate the origin of these structures and to better define the physical parameters relevant to this phenomenon. The 
remote sensing observations combined with in situ observations performed in the future on board the ESA's Solar Orbiter will be extremely helpful to draw a conclusive picture of this intriguing heliospheric phenomenon.

\section{Appendix A}

\section{The Grad-Shafranov reconstruction}

For a quasi-stationary two-dimensional magnetic field structure, in a reference frame moving with the structure, the equilibrium equation can be written as the plane GS equation:

$\nabla^{2} A=\frac{\partial^{2} A}{\partial x^{2}}+\frac{\partial^{2} A}{\partial y^{2}}=-\mu_{0} \frac{d}{d A}\left(p+\frac{B_{z}^{2}}{2 \mu_{o}}\right)$

where $z$ is along the invariant direction and $A$ is the $z$ component of the vector potential. The right-hand side of the GS equation is the first derivative of the transverse pressure $P_{\mathrm{t}}=\left(p+\frac{B_{\mathrm{z}}^{2}}{2 \mu_{\mathrm{o}}}\right)$, which, within these structures, is a function of $A$ alone.

The magnetic field vector is expressed as $\boldsymbol{B}=$ $\left(\frac{\partial A}{\partial y} ;-\frac{\partial A}{\partial x} ; B_{\mathrm{Z}}(x, y)\right)$. By choosing $x$ along the velocity of the structure $-V_{0}$, the $x$ axis represents the path along which the spacecraft moves through the structure. In this way, the vector potential along the $x$ axis can be obtained as $A(x, 0)=-V_{0} \int_{0}^{t} B_{\mathrm{y}}\left(t^{\prime}\right) d t^{\prime}$.

The velocity of the structure can be obtained with the deHoffmann-Teller (HT) analysis (Khrabrov and Sonnerup, 1998). A good correlation among the electric fields $-V \times B$ and $-V_{\mathrm{HT}} \times B$ implies that the structure is quasi-stationary, and $V_{\mathrm{HT}}$ can be assumed as the velocity of the structure.

An optimization procedure, based on the requirement that $P_{\mathrm{t}}$ is a single-value function of $A$, can be used to evaluate the orientation of the invariant axis (Hu and Sonnerup, 2002). In this method, each possible orientation of the invariant axis $(z)$ in the three-dimensional space is considered, and, for each orientation, the $x$ axis is along the projection of $-V_{\mathrm{HT}}$ in the plane perpendicular to $z$, and the $y$ axis completes the orthogonal triad. For each trial reference, the transverse pressure $P_{\mathrm{t}}(x, 0)$ and the vector potential $A(x, 0)$ are calculated, and a residual RES proportional to the deviation of $P_{\mathrm{t}}(x, 0)$ versus a single-value function of $A(x, 0)$ is evaluated (Eq. 5 of $\mathrm{Hu}$ and Sonnerup, 2002). The optimal orientation of the structure is given along the direction corresponding to the minimum RES.

Once the optimal reference of the structure is determined, the $P_{\mathrm{t}}(A)$ is fitted with a proper analytical function $P_{\mathrm{t}}\left(A\left(x_{i}, 0\right)\right)$ so that the right-hand side of the GS equation can be evaluated. The GS equation is then integrated numerically in the reconstruction plane as a Cauchy problem by using the spacecraft data as the initial values (see e.g. Hau and Sonnerup, 1999).

The deviations of a reconstructed structure from the GS assumptions can be quantitatively estimated with the fitting residual $R_{\mathrm{f}}$ (Hu et al., 2004) defined as

$R_{\mathrm{f}}=\frac{\sqrt{\frac{1}{N} \sum_{i}\left[P_{\mathrm{t}}\left(x_{i}, 0\right)-P_{\mathrm{t}}\left(A\left(x_{i}, 0\right)\right)\right]^{2}}}{\max \left(P_{\mathrm{t}}(x, 0)\right)-\min \left(P_{\mathrm{t}}(x, 0)\right)}$.

Acknowledgements. This work was partially supported by the Agenzia Spaziale Italiana, contract ASI/INAF I/013/12/0.

Topical Editor I. A. Daglis thanks C. Cid and one anonymous referee for their help in evaluating this paper.

\section{References}

Bavassano, B. and Bruno, R.: Evidence of local generation of Alfvénic turbulence in the solar wind, J. Geophys Res., 94, 11977-11982, 1989.

Borovsky, J. E.: Flux tube texture of the solar wind: Strands of the magnetic carpet at $1 \mathrm{AU}$ ?, J. Geophys Res., 113, A08110, doi:10.1029/2007JA012684, 2008.

Bruno, R., Carbone, V., Veltri, P., Pietropaolo, E., and Bavassano, B.: Identifying intermittency events in the solar wind, Planet. Space Sci., 49, 1201-1210, 2001.

Bruno, R., Pietropaolo, E., Servidio, S., Greco, A., Matthaeus, W. H., D’Amicis, R., Sorriso-Valvo, L., Carbone, V., Balogh, A., Bavassano, B.: Spatial and Temporal Analysis of Magnetic Helicity in the Solar Wind, AGU Fall Meeting Abstracts, A6, 2008.

Chollet, E. E. and Giacalone, J.: Multispacecraft Analysis of Energetic Ion Flux Dropouts, Astrophys. J., 688, 1368-1373, 2008.

Chuychai, P., Ruffolo, D., Matthaeus, W. H., Meechai, J.: Trapping and Diffusive Escape of Field Lines in Two-Component Magnetic Turbulence, Astrophys. J., 659, 1761-1776, 2007.

Dasso, S., Mandrini, C. H., Démoulin, P., and Luoni, M. L.: A new model-independent method to compute magnetic helicity in magnetic clouds, Astron. Astrophys., 455, 349-359, 2006.

Dröge, W. J.: Solar Particle Transport in a Dynamical Quasi-linear Theory, Astrophys. J., 589, 1027-1039, 2003.

Dröge, W. and Kartavykh, J.: Testing Transport Theories with Solar Energetic Particles, Astrophys. J., 693, 69-74, 2009.

Giacalone, J. and Jokipii, J. R.: The Transport of Cosmic Rays across a Turbulent Magnetic Field, Astrophys. J., 520, 204-214, 1999.

Giacalone, J., Jokipii, J. R., and Mazur, J. E.: Small-scale Gradients and Large-scale Diffusion of Charged Particles in the Heliospheric Magnetic Field, Astrophys. J., 532, L75-L78, 2000.

Gosling, J. T., Skoug, R. M., McComas, D. J., and Mazur, J. E.: Correlated Dispersionless Structure in Suprathermal Electrons and Solar Energetic Ions in the Solar Wind, Astrophys. J., 614, 412419, 2004.

Hau, L.-N. and Sonnerup, B. U. Ö.: Two-dimensional coherent structures in the magnetopause: Recovery of static equilibria from single-spacecraft data, J. Geophys Res., 104, 6899-6918, 1999.

He, J., Marsch, E., Tu, C., Yao, S., and Tian, H.: Possible Evidence of Alfvén-cyclotron Waves in the Angle Distribution of Magnetic Helicity of Solar Wind Turbulence, Astrophys. J., 731, 85, doi:10.1088/0004-637X/731/2/85, 2011.

$\mathrm{Hu}$, Q. and Sonnerup, B. U. Ö.: Reconstruction of magnetic flux ropes in the solar wind, Geophys Res. Lett., 28, 467-470, 2001. 
Hu, Q. and Sonnerup, B. U. Ö.: Reconstruction of magnetic clouds in the solar wind: Orientations and configurations, J. Geophys Res., 107, 1142, doi:10.1029/2001JA000293, 2002.

Hu, Q., Smith, C. W., Ness, N. F., and Skoug, R. M.: Double fluxrope magnetic cloud in the solar wind at $1 \mathrm{AU}$, Geophys Res. Lett., 30, 38-1-38-4, 2003.

Hu, Q., Smith, C. W., Ness, N. F., and Skoug, R. M.: Multiple flux rope magnetic ejecta in the solar wind, J. Geophys Res., 109, A03102, doi:10.1029/2003JA010101, 2004.

Jokipii, J. R.: Cosmic-Ray Propagation. I. Charged Particles in a Random Magnetic Field, Astrophys. J., 146, 480-487, 1966.

Jokipii, J. R. and Parker, E. N.: Random Walk of Magnetic Lines of Force in Astrophysics, Phys. Rev. Lett., 21, 44-47, 1968.

Khrabrov, A. V. and Sonnerup, B. U. Ö.: DeHoffmann-Teller Analysis, Analysis Methods for Multi-Spacecraft Data, edited by: Paschmann, G. and Daly, P., ISSI Scientific Reports Series, 1, 221-248, 1998.

Krittinatham, W. and Ruffolo, D.: Drift Orbits of Energetic Particles in an Interplanetary Magnetic Flux Rope, Astrophys. J., 704, 831-841, 2009.

Li, G.: Flux tubes in the fast and slow solar wind, in: Turbulence and Nonlinear Processes in Astrophysical Plasmas, edited by: Shaikh, D. and Zank, G. P., American Institute of Physics Conference Series, 932, p. 26-31, doi:10.1063/1.2778941, 2007.

Li, G.: Identifying current-sheet-like structures in the solar wind, Astrophys. J. Lett., 672, L65, doi:10.1086/525847, 2008.

Mason, G. M., Gold, R. E., Krimigis, S. M., Mazur, J. E., Andrews, G. B., Daley, K. A., Dwyer, J. R., Heuerman, K. F., James, T. L., Kennedy, M. J., Lefevere, T., Malcolm, H., Tossman, B., and Walpole, P. H.: The Ultra-Low-Energy Isotope Spectrometer (ULEIS) for the ACE spacecraft, Space Sci. Rev., 86, 409-448, 1998.

Masson, S., Démoulin, P., Dasso, S., and Klein, K.-L.: The interplanetary magnetic structure that guides solar relativistic particles, Astron. Astrophys., 538, A32, doi:10.1051/00046361/201118145, 2012.

Mazur, J. E., Mason, G. M., Dwyer, J. R., Giacalone, J., Jokipii, J. R., and Stone, E. C.: Interplanetary Magnetic Field Line Mixing Deduced from Impulsive Solar Flare Particles, Astrophys. J. Lett., 532, L79-L82, 2000.

McComas, D. J., Bame, S. J., Barker, P., Feldman, W. C., Phillips, J. L., Riley, P., and Griffee, J. W.: Solar Wind Electron Proton Alpha Monitor (SWEPAM) for the Advanced Composition Explorer, Space Sci. Rev., 86, 563-612, 1998.

Moldwin, M. B., Ford, S., Lepping, R., Slavin, J., and Szabo, A.: Small-scale magnetic flux ropes in the solar wind, Geophys Res. Lett., 27, 57-60, 2000.

Podesta, J. J. and Gary, S. P.: Magnetic Helicity Spectrum of Solar Wind Fluctuations as a Function of the Angle with Respect to the Local Mean Magnetic Field, Astrophys. J., 734, 15, doi:10.1088/0004-637X/734/1/15, 2011.
Pommois, P., Zimbardo, G., and Veltri, P.: Energetic particle transport in anisotropic magnetic turbulence, Adv. Space Res., 35 647-652, 2005.

Qin, G. and Li, G.: Effect of Flux Tubes in the Solar Wind on the Diffusion of Energetic Particles, Astrophys. J., 682, L129-L132, 2008.

Reames, D. V.: Coronal element abundances derived from solar energetic particles, Adv. Space Res., 14, 177-180, 1994.

Reames, D. V.: Particle acceleration at the Sun and in the heliosphere, Space Sci. Rev., 90, 413-491, 1999.

Ruffenach, A., Lavraud, B., Owens, M. J.,Sauvaud, J.-A., Savani, N. P., Rouillard, A. P., Démoulin, P., Foullon, C., Opitz, A., Fedorov, A., Jacquey, C. J., Génot, V., Louarn, P., Luhmann, J. G., Russell, C. T., Farrugia, C. J., and Galvin, A. B.: Multispacecraft observation of magnetic cloud erosion by magnetic reconnection during propagation, J. Geophys Res., 117, A09101, doi:10.1029/2012JA017624, 2012.

Ruffolo, D., Matthaeus, W. H., and Chuychai, P.: Trapping of Solar Energetic Particles by the Small-Scale Topology of Solar Wind Turbulence, Astrophys. J., 597, L169-L172, 2003.

Ruiz, M. E., Dasso, S., Matthaeus, W. H., Marsch, E., and Weygand, J. M.: Aging of anisotropy of solar wind magnetic fluctuations in the inner heliosphere, J. Geophys Res., 116, A10102, doi:10.1029/2011JA016697, 2011.

Smith, C. W., L'Heureux, J., Ness, N. F., Acuña, M. H., Burlaga, L. F., and Scheifele, J.: The ACE Magnetic Fields Experiment, Space Sci. Rev., 86, 613-632, 1998.

Sonnerup, B. U. Ö. and Guo, M.: Magnetopause transects, Geophys Res. Lett., 23, 3679-3682, 1996.

Telloni, D., Bruno, R., D'Amicis, R., Pietropaolo, E., and Carbone, V.: Wavelet Analysis as a Tool to Localize Magnetic and Cross-helicity Events in the Solar Wind, Astrophys. J., 751, doi:10.1088/0004-637X/751/1/19, 2012.

Thieme, K. M., Schwenn, R., and Marsch, E.: Are structures in high-speed streams signatures of coronal fine structures?, Adv. Space Res., 9, 127-130, 1989.

Trenchi, L., Bruno, R., Telloni, D., D’amicis, R., Marcucci, M. F., Zurbuchen, T. H., and Weberg, M.: Solar Energetic Particle Modulations Associated with Coherent Magnetic Structures, Astrophys. J., 770, doi:10.1088/0004-637X/770/1/11, 2013.

Tu, C.-Y. and Marsch, E.: Transfer equations for spectral densities of inhomogeneous MHD turbulence, J. Plasma Phys., 44, 103122, 1990.

Tu, C.-Y. and Marsch, E.: A model of solar wind fluctuations with two components - Alfvén waves and convective structures, J. Geophys Res., 98, 1257-1276, 1993.

Zimbardo, G., Pommois, P., and Veltri, P.: Visualizing Particle Transport Across Magnetic Flux Tubes in Anisotropic Magnetic Turbulence, IEEE Trans. Plasma Sci., 36, 1114-1115, 2008. 